\title{
Author Correction: Time-lapse imagery and volunteer classifications from the Zooniverse Penguin Watch project
}

Fiona M. Jones ${ }^{1}$, Campbell Allen ${ }^{2}$, Carlos Arteta ${ }^{3}$, Joan Arthur ${ }^{2}$, Caitlin Black ${ }^{1}$, Louise M. Emmerson ${ }^{4}$, Robin Freeman ${ }^{5}$, Greg Hines ${ }^{2}$, Chris J. Lintott ${ }^{2}$, Zuzana Macháčková Grant Miller ${ }^{2}$, Rob Simpson ${ }^{6}$, Colin Southwell ${ }^{4}$, Holly R. Torsey ${ }^{2}$, Andrew Zisserman ${ }^{3}$ \& Tom Hart ${ }^{1}$

Correction to: Scientific Data https://doi.org/10.1038/sdata.2018.124, published online 26 June 2018

Following publication, it was discovered that two of the datasets deposited to Dryad to accompany this Data Descriptor were duplicates. The affected folders were named PETEc2014 and PETEd2013. As a result of this, there are a number of instances in the text where the total number of included images, the number of cameras that they were captured by, and the number of metadata files is stated incorrectly. These errors and the corresponding correct values are listed below:

- In the Abstract and Data Records section it is incorrectly stated that images were captured from 15 cameras. The correct number is 14 .

- In the Abstract, Data Records section and Figure 4 legend it is incorrectly stated that 73,802 images were captured in total. The correct number of images is 63,070.

- In Table 4 and its accompanying footnote it is also incorrectly stated that 73,802 images were captured in total. The correct number of images is 63,070.

- Tables 4, 5 and 6 mention the duplicate PETEd2013 folder. This has now been removed from the Dryad dataset.

- In the Data Records section it is incorrectly stated that images are grouped into 23 separate folders, with 34 corresponding 'consensus click' folders and 34 metadata folders. Since the PETEd 2013 image folder and associated citizen science files have been removed, the correct number of image folders is 22 , and there are 32 corresponding 'consensus click' and metadata files.

Please note that the PETEc images used for the Technical Validation were randomly selected from the PETEc2013 dataset (not PETEc2014), and the PETEd2013 citizen science classifications were not incorporated into this analysis.

A new version of the dataset has been deposited to Dryad, from which the duplicate image folder (PETEd2013) and citizen science data files (PETEd2013a_concl, PETEd2013b_concl, PETEd2013a_metadata and PETEd2013b_metadata) have been removed. An accompanying version history and Erratum notice have been added to account for the change. The new version can be accessed via the existing citation in the accompanying Data Descriptor, or directly at https://doi.org/10.5061/dryad.vv36g.2.

${ }^{1}$ Department of Zoology, University of Oxford, South Parks Road, Oxford OX1 3PS, UK. ${ }^{2}$ Zooniverse, Department of Physics, University of Oxford, Denys Wilkinson Building, Keble Road, Oxford OX1 3RH, UK. ${ }^{3}$ Department of Engineering Science, University of Oxford, Parks Road, Oxford OX1 3PJ, UK. ${ }^{4}$ Department of the Environment and Energy, Australian Antarctic Division, 203 Channel Highway, Kingston, Tasmania 7050, Australia. ${ }^{5}$ nnstitute of Zoology, Wellcome Building, Outer Circle, Regent's Park, London NW1 4RY, UK. ${ }^{6}$ Google UK, Belgrave House, 76 Buckingham Palace Road, London SW1W 9TQ, UK. Correspondence and requests for materials should be addressed to F.M.J. (email: fiona.jones@zoo.ox.ac.uk)orT.H. (email: tom.hart@zoo.ox.ac.uk) 
(1) Open Access This article is licensed under a Creative Commons Attribution 4.0 International License, which permits use, sharing, adaptation, distribution and reproduction in any medium or format, as long as you give appropriate credit to the original author(s) and the source, provide a link to the Creative Commons license, and indicate if changes were made. The images or other third party material in this article are included in the article's Creative Commons license, unless indicated otherwise in a credit line to the material. If material is not included in the article's Creative Commons license and your intended use is not permitted by statutory regulation or exceeds the permitted use, you will need to obtain permission directly from the copyright holder. To view a copy of this license, visit http://creativecommons.org/licenses/by/4.0/.

(c) The Author(s) 2019 